\title{
Reply to comment on: Godsland IF, Jeffs JA, Johnston DG (2004) Loss of beta cell function as fasting glucose increases in the non-diabetic range. Diabetologia 47:1157-1166
}

Received: 28 June 2005 / Accepted: 30 June 2005 / Published online: 26 August 2005

(C) Springer-Verlag 2005

To the Editor: As we stated in our original paper [1], our intention was to define the level of glycaemia at which pancreatic insulin secretion begins to decline. We were careful throughout to emphasise that we were concerned not with causal relationships but with identifying the principal accompaniments of deteriorating fasting plasma glucose (FPG) levels in the sub-diabetic range. We agree with Abdul-Ghani and DeFronzo that the decline in IVGTT first-phase insulin secretion that accompanies the rise in FPG does not necessarily indicate a causal relationship [2]. Nevertheless, in our study, the decline in first-phase pancreatic insulin secretion was by far the most striking feature that accompanied increasing glucose levels. As AbdulGhani and DeFronzo affirm, the important aspect of this decline was that it occurred within ranges of glucose concentrations hitherto considered normal.

In drawing attention to glucotoxicity and the sensitivity of pancreatic insulin secretion to rising glucose levels, Abdul-Ghani and DeFronzo raise an important mechanistic possibility. This does, however, pose a potential 'chicken and egg' problem, since glucose concentrations would have to rise for there to be deficient pancreatic insulin secretion, but there might have to be deficient insulin secretion for glucose concentrations to rise. Which comes first? One possibility, which they allude to, is that it is not decreasing insulin secretion that sets glucose levels rising

\footnotetext{
I. F. Godsland · D. G. Johnston

Department of Endocrinology and Metabolic Medicine,

Division of Medicine, Faculty of Medicine,

Imperial College London,

London, UK

I. F. Godsland ( $\square)$

Wynn Reader in Human Metabolism,

Department of Endocrinology and Metabolic Medicine,

Division of Medicine, Faculty of Medicine,

Imperial College London,

St Mary's Hospital,

Mint Wing 2nd Floor,

London, W2 1PG, UK

e-mail: i.godsland@imperial.ac.uk

Tel.: +44-207-8866573

Fax: $+44-207-8861790$
}

but decreasing insulin sensitivity. Our finding that there was little change in insulin sensitivity with increasing FPG appears contrary to the weight of evidence in this respect.

Abdul-Ghani and DeFronzo raise the possibility that our IVGTT protocol was not sensitive enough to detect impaired insulin sensitivity [2]. That this is not the case is illustrated by our previous publications, which attest to the ability of our protocol to detect diminished insulin sensitivity in a variety of circumstances and to detect significant associations with metabolic correlates of insulin sensitivity at a strength equivalent to that seen with other protocols and methodologies (see [3-6] for examples). Abdul-Ghani and DeFronzo also question the relevance of conclusions regarding pancreatic insulin secretion based on the non-physiological intravenous route of administration. However, they point out that, consistent with our results [1], in their own studies using the OGTT [7] there was a decline in the OGTT plasma insulin response with increasing FPG at levels $>5.6 \mathrm{mmol} / \mathrm{l}$. It is also noteworthy that in the study of Ozaki et al. of 8,923 men and women in Japan, in which OGTT insulin concentrations at 30 and 120 min provided indices of early- and late-phase insulin secretion, relationships were noted between plasma insulin concentrations and FPG levels in the range $4.4-8.3 \mathrm{mmol} / \mathrm{l}$ that were similar to those we distinguished between pancreatic insulin secretion and FPG [8].

With regard to the apparent lack of association between insulin sensitivity and FPG in our study, the critical point to note is that, as stated in the table legend, the data we reported in Table 2 was standardised for age and BMI. As shown in Table 4 of our paper, we did, in fact, identify a significant association between increasing FPG and declining insulin sensitivity, but, in contrast to the various insulin secretion measures, this association ceased to be significant in multiple linear regression analysis when age and BMI were taken into account. The key covariate in this loss of significance appeared to be BMI.

Interestingly, the discrepancy between the effect of standardisation for BMI on the associations of FPG with both insulin sensitivity and insulin secretion might provide a clue as to how the cycle of rising FPG concentrations and 
declining pancreatic insulin secretion becomes established. Increasing adiposity is associated with increasing circulating levels of NEFAs and cytokines, which can adversely affect both insulin sensitivity and pancreatic insulin secretion [9]. As mentioned, insulin secretion is also adversely affected by increasing glucose levels, but this may not be the case for insulin sensitivity. Increasing adiposity could, therefore, set in motion a self-sustaining cycle of increasing plasma glucose levels and decreasing insulin secretion, with declining insulin sensitivity as a secondary accompaniment. Consequently, standardisation for BMI eliminates the association between increasing FPG and decreasing insulin sensitivity, but not that between increasing FPG and decreasing insulin secretion. This is, of course, a hypothetical possibility that will need further investigation. It could, however, explain how our standardisation for BMI could eliminate the significant association between FPG and insulin sensitivity, but not that between FPG and insulin secretion.

\section{References}

1. Godsland IF, Jeffs JA, Johnston DG (2004) Loss of beta cell function as fasting glucose increases in the non-diabetic range. Diabetologia 47:1157-1166
2. Abdul-Ghani MA, DeFronzo RA (2005) Comment to Godsland IF, Jeffs JA, Johnston DG (2004) Loss of beta cell function as fasting glucose increases in the non-diabetic range. Diabetologia DOI 10.1007/s00125-005-1883-3

3. Godsland IF, Walton C, Felton C, Proudler AJ, Patel A, Wynn $\mathrm{V}$ (1992) Insulin resistance, secretion and metabolism in users of oral contraceptives. J Clin Endocrinol Metab 74:64-70

4. Ley C, Swan J, Godsland I, Walton C, Crook D, Stevenson J (1994) Insulin resistance, lipids, body fat and coagulation factors in males with suspected angina and normal or abnormal coronary angiograms. J Am Coll Cardiol 23:377-383

5. Godsland IF, Crook D, Walton C, Wynn V, Oliver MF (1992) Influence of insulin resistance, secretion, and clearance on serum cholesterol, triglycerides, lipoprotein cholesterol, and blood pressure in healthy men. Arterioscler Thromb 12:1030 1035

6. Godsland IF, Crook D, Proudler AJ, Stevenson JC (2005) Hemostatic risk factors and insulin sensitivity, regional body fat distribution and the metabolic syndrome. J Clin Endocrinol Metab 90:190-197

7. Gastaldelli A, Ferrannini E, Miyazaki Y, Matsuda M, DeFronzo RA (2004) Beta-cell dysfunction and glucose intolerance: results from the San Antonio metabolism (SAM) study. Diabetologia 47:31-39

8. Ozaki K, Okubo M, Mori H, Mito K, Hara H, Kohno N (2002) Decreased insulin secretion and dyslipidemia coexist in subjects with impaired fasting glucose. Diabetes Res Clin Pract 55:159-164

9. Stumvoll M, Goldstein BJ, van Haeften TW (2005) Type 2 diabetes: principles of pathogenesis and therapy. Lancet 365: $1333-1346$ 